\title{
RECONFIGURABLE FLIGHT CONTROL USING NONLINEAR DYNAMIC INVERSION WITH A SPECIAL ACCELEROMETER IMPLEMENTATION
}

\author{
Barton J. Bacon and Aaron J. Ostroff \\ NASA Langley Research Center \\ Hampton, VA
}

\begin{abstract}
This paper presents an approach to on-line control design for aircraft that have suffered either actuator failure, missing effector surfaces, surface damage, or any combination. The approach is based on a modified version of nonlinear dynamic inversion. The approach does not require a model of the baseline vehicle (effectors at zero deflection), but does require feedback of accelerations and effector positions. Implementation issues are addressed and the method is demonstrated on an advanced tailless aircraft. An experimental simulation analysis tool is used to directly evaluate the nonlinear system's stability robustness.
\end{abstract}

\section{Introduction}

Reconfiguration in flight concerns the ability of the feedback system to redesign itself, in the presence of actuator failure, missing surfaces, and wing damage, to maintain stability and some acceptable level of handling qualities. A reconfigurable control law is generally the integration of at least three important elements:

1)failure detection and isolation to determine which surface is no longer useful, 2)on-line parameter identification to provide a model of the damaged vehicle, and 3)on-line control design which utilizes the information from the other two elements to reestablish control. This paper deals exclusively with the third element: specifically, developing an on-line control design methodology that is robust to information errors obtained from the other two elements.

Over the past decade, the literature concerning the on-line design portion of reconfigurable controls has been dominated by two concepts: dynamic inversion $(\mathrm{DI})^{1}$ and receding horizon optimal (RHO) ${ }^{2}$ control.

This is not surprising since both can be readily modified to handle changing nonlinear dynamics with updates to the control's required onboard model. Also, both concepts include provisions for obtaining desired closed-loop dynamics (either explicitly or implicitly) to

Copyright $(2000$ by the American Institute of Aeronautics and Astronautics, Inc. No copyright is asserted in the United States under Title 17, U.S. Code. The U.S. Government has a royalty-free license to exercise all rights under the copyright claimed herein for Governmental Purposes. All other rights are reserved by the copyright owner satisfy handling qualities. Unfortunately, both concepts tend to be sensitive to onboard model error.

RHO was used in the Self-Designing Flight Control (SDFC) ${ }^{3}$ which matured in Lockheed-Martin's approach to the Reconfigurable Control For Tailless Fighter Aircraft (RESTORE) program ${ }^{4}$. In SDFC, the LQ control stability robustness guarantees of RHO were exploited to successfully reconfigure the VISTA/F-16 on landing subject to a simulated missing left tail surface. In RESTORE, the full predictive advantage of RHO was utilized to better anticipate the desired plant response producing responses that were more decoupled and closer to the desired ones.

Some challenges from SDFC persist for RHO. Specifically, the implemented Level 1 models did not always result in Level 1 flying qualities. This problem was not observed in ground-based simulation. Contributing factors could be sensitivity to onboard model errors in flight and the lack of guidelines for setting or adjusting on-line the algorithm's cost function weightings, penalty terms, and finite time horizon. These factors all influence how well the control follows the reference model. In contrast, DI avoids the problem of manipulating cost functions, but not necessarily the problem of sensitivity due to onboard model error.

The DI-based, Self-Repairing Flight Control System (SRFC) ${ }^{5}$, for example, achieved on-line control redesign using onboard models of both the nominal and the current/damaged vehicle to detect acceleration mismatch. This mismatch drove a pseudo inverse allocator to recover the commanded acceleration of the nominal flight controller. Augmented to an existing F-15 controller, the flight experiment demonstrated that this form of dynamic inversion was sensitive to model errors not only in the current model but in the nominal model which was based on a mature aerodynamic database of the F-15.

A similar issue surfaced when the Intelligent Flight Control (IFC) used $\mathrm{DI}^{6.7 .8}$ as an explicit model follower. In the IFC structure, dynamic inversion was not applied to the actual vehicle but to an onboard model. Plant input and desired output reference signals from this onboard system were fed to a closed-loop feedback system involving the actual vehicle to robustly produce the desired response. IFC was not robust enough, however, in that it failed to yield the desired

1

American Institute of Aeronautics and Astronautics 
longitudinal response on the ACTIVE/F 15 due to onboard model error produced by the control's neural net. The net was trained on a database for a vehicle with square thrust vectoring nozzles. The test vehicle had been recently upgraded to round nozzles.

The issue of model error and dynamic inversion in on-line control design was revisited in Boeing's approach to RESTORE' ${ }^{9}$. A different explicit model following control structure was selected where dynamic inversion was applied directly to the vehicle. An online neural network, based on the work of Kim and Calise ${ }^{10}$, adaptively regulated the error in the plant inversion to yield the desired rate response of select control variables. In truth, the net was a short term memory device that modeled the inversion error. If the optimal fit (not necessarily obtained on-line) satisfied a Lyapunov-based constraint ${ }^{10,11,12}$, the controller could stabilize the vehicle without immediate intervention from parameter identification. The optimal fit is determined by the net's structure, i.e. the number/type of basis functions and weightings. For the failures considered in RESTORE, the number of weightings required in the pitch, roll, and yaw channels were 18 , 72 , and 72 respectively ${ }^{13}$. For failures not considered, no mechanism was offered to adapt the structure, i.e. complexity, of the neural net to model different sets of inversion errors. This is one approach to desensitize dynamic inversion to model uncertainty. Another is the approach taken in this paper which reformulates dynamic inversion to be less dependent on the onboard model.

The proposed approach to desensitize dynamic inversion for on-line control design is an extension of Smith's approach for standard flight control ${ }^{14}$ to address reconfiguration, various response types, and the incorporation of handling qualities. Similar features include the absence of an onboard model of the baseline aircraft (no control deflections) and the required feedback of the accelerations and effector positions. Differences include a proposed strategy for dealing with nonaffine controls--no inverse mapping via neural nets required $^{10}$. Here, a special implementation of accelerometers provides the required accelerations with considerable redundancy to accommodate sensor failure. The proposed control strategy is most closely aligned with SRFC but without the required onboard models. The proposed control is an implicit model following control requiring only the system's control derivatives. Robustness results, using an advanced tailless aircraft with multiple innovative control effectors ${ }^{15,16}$, will show that the accuracy of these control derivatives are not stringent.

To provide a clear exposition of the method, the paper is organized as follows. First, a modified version of dynamic inversion is developed for on-line control design. Implementation issues associated with obtaining the required accelerations, generating the vehicles control derivatives, and promoting the desired flying qualities follow. Several examples are then offered to demonstrate the efficacy of this approach to reconfiguration. Lastly, an experimental simulation analysis tool is used to directly evaluate the nonlinear system's stability robustness.

\section{Modified Dynamic Inversion for On-line Control Design}

In this section, a general development of the online control design is offered. Unlike most of the other dynamic inversion-based on-line design techniques mentioned above, the method pursued here is implicit. The desired dynamics of the closed-loop system do not reside in some explicit model to be followed but result when the feedback loops are closed. Implicit dynamic inversion has been used previously in DI-based flight control systems. ${ }^{1.17,18}$ Due to the demands placed on the on-line control design (i.e., unforeseen rapidly changing plant dynamics due to failure occurring possibly during complex maneuvering) the form developed here will be much less dependent on the control's onboard model.

For this discussion, let $x$ denote the state of the aircraft whose motion is governed by

$$
\dot{x}=F(x, \delta)=f(x)+g(x, \delta)
$$

where the $\operatorname{dim}(\delta)>3$. The control objective here is to obtain $\delta$ such that three state-dependent control variables corresponding to the longitudinal, lateral, and directional axes,

$$
y=\left[\begin{array}{lll}
y_{\text {lon }} & y_{\text {lat }} & y_{\text {dir }}
\end{array}\right]^{T}=h(x)
$$

have some desired behavior under both nominal and failed conditions. The desired behavior of $y$ to some commanded input $y_{c}$ is typically defined by

$$
\dot{y}=f_{\text {des }}\left(y, y_{c}\right)
$$

yielding low-order responses that are decoupled along axes and compliant with military specifications ${ }^{19}$.

A dynamic inversion approach to this problem involves constructing a control of the form

$$
\delta=k\left(x, \dot{y}_{\text {des }}\right)
$$

to realize some desired control variable rate $\dot{y}=\dot{y}_{\text {des }}$ when substituted into equation 1 subject to equation 2. To realize desired closed-loop dynamics of equation 3 , the control in its implicit form is

$$
\delta=k\left(x, f_{\text {des }}\left(h(x), y_{c}\right)\right) \text {. }
$$

If the input mapping $g(x, \delta)$ can be expressed as

$$
g(x, \delta)=G(x) \delta
$$


the dynamic inversion control, subject to minimizing $\delta^{T} W^{-1} \delta$, is

$\delta=W\left(h_{x} G\right)^{T}\left[h_{x} G W\left(h_{x} G\right)^{T}\right]^{-1}\left(\dot{y}_{\text {des }}-h_{x} f\right)$

where $h_{x}=\partial h / \partial x$ and $W$ is a diagonal weighting matrix. The dependency on $x$ has been dropped for notational convenience.

The control in equation 7 is clearly dependent on the onboard nonlinear model described by $f$ and $G$. Such a model was required for standard flight controls. ${ }^{17,18}$ For on-line control design, the model would have to be updated in flight. Due to the model's form, an update requires more information than just the stability and control derivatives identified online. ${ }^{20}$

The problem is that the stability derivatives do not define the elements of $f$. Note, the control derivatives are the elements of $G$. The elements of $f$ are defined if the on-line parameter identification also identifies a bias $^{3}$, or a model of the form

$\dot{x} \equiv A x+G \delta+$ bias

with

$f \cong A x+$ bias.

Here, $x$ is the actual state (not a perturbation) and the elements of $A$ and $G$, are the stability and control derivatives, respectively. A key difficulty for dynamic inversion is the onboard storage of the nominal bias and the required bias corrections which typically depend on all the elements of $x$.

Another problem with equation 7 for on-line control design concerns the system's actual control mapping. The required mapping, $G \delta$, is actually the nonlinear control mapping $g(x, \delta)$ rendering the pseudo inverse solution in (7) inappropriate. While some have proposed neural nets ${ }^{10}$ to get around this problem, there is a much easier procedure using a modified approach to dynamic inversion that eliminates all the problems cited.

To develop the modified form, consider the equations of motion as they actually appear with a nonlinear control mapping

$$
F(x, \delta)=f(x)+g(x, \delta)=\dot{x} .
$$

A standard Taylor series expansion provides the following first-order approximation of $F(x, \delta)$ for $x$ and $\delta$ in the neighborhood of $\left[x_{0}, \delta_{o}\right]$

$$
\begin{aligned}
& F(x, \delta) \cong f\left(x_{o}\right)+g\left(x_{o}, \delta_{o}\right)+ \\
& \left.\frac{\partial}{\partial x}(f(x)+g(x, \delta))\right|_{x=x_{o}, \delta=\delta_{o}}\left(x-x_{o}\right)+ \\
& \left.\frac{\partial}{\partial \delta}(g(x, \delta))\right|_{x=x_{o}, \delta=\delta_{o}}\left(\delta-\delta_{o}\right)
\end{aligned}
$$

Let $x_{o}$ and $\delta_{o}$ denote some previous state and control from the recent past. The corresponding state rate $\dot{x}_{o}$ satisfies

$$
\dot{x}_{o} \equiv f\left(x_{o}\right)+g\left(x_{o}, \delta_{o}\right) \text {. }
$$

Using the standard linear definition,

$$
\begin{aligned}
& A_{o}=\left.\frac{\partial}{\partial x}(f(x)+g(x, \delta))\right|_{x=x_{o}, \delta=\delta_{o}} \\
& B_{o}=\left.\frac{\partial}{\partial \delta}(g(x, \delta))\right|_{x=x_{o}, \delta=\delta_{o}}
\end{aligned}
$$

with partials evaluated at points $\left(x_{o}, \delta_{o}\right)$ on the state/control trajectory,

$$
F(x, \delta)=\dot{x} \cong \dot{x}_{o}+A_{o}\left(x-x_{o}\right)+B_{o} \Delta \delta
$$

in the neighborhood of $\left[x_{o}, \delta_{o}\right]$ where $\Delta \delta=\delta-\delta_{o}$.

With regard to dynamic inversion control, $y=h(x)$ and

$$
\dot{y}=\frac{\partial h(x)}{\partial x} \dot{x} \cong h_{x}\left(\dot{x}_{o}+A_{o}\left(x-x_{o}\right)+B_{o} \Delta \delta\right) .
$$

As before, a minimum norm solution provides a $\Delta \delta$ so $\dot{y}_{d e s}=\dot{y}$ subject to minimizing $\Delta \delta^{T} W^{-1} \Delta \delta$

$$
\begin{gathered}
\Delta \delta=W\left(h_{x} B_{o}\right)^{T}\left[h_{x} B_{o} W\left(h_{x} B_{o}\right)^{T}\right]^{-1}\left(\dot{y}_{d e s}-\right. \\
\left.\left(h_{x} \dot{x}_{o}+h_{x} A_{o}\left(x-x_{o}\right)\right)\right)
\end{gathered}
$$

With a sufficient control update rate, $x$ approaches $x_{o}$ and the control becomes

$$
\Delta \delta=W\left(h_{x} B_{o}\right)^{T}\left[h_{x} B_{o} W\left(h_{x} B_{o}\right)^{T}\right]^{-1}\left(\dot{y}_{\text {des }}-h_{x} \dot{x}_{o}\right)
$$

with $\delta=\delta_{o}+\Delta \delta$. It is important to note that in this formulation $\dot{x}_{o}$ is based on the effector position $\delta_{o}$ and $\Delta \delta$ is the commanded change to that position in response to $\dot{y}_{\text {des }}$. Hence, sensed positions of the effectors are a must. The modified dynamic inversion inner loop is shown in figure 1 . Note, the existing $\delta_{o}$ that corresponds to $\dot{x}_{o}$ is taken from the output of the actuator. The weighting $W$ consists of the effector's rate limit emphasizing the faster controls in the minimum norm solution.

The desired dynamics defined by equation 3 along with equation 17 define the proposed online control design method. Note, the control requires the vehicle's control derivatives, $B_{o}$, either in table lookup form or some functional approximation form. The control does not require $f(x)$ or $g(x, \delta)$. Changes in $f(x)$ are reflected in the measurement of $\dot{x}_{0}$. The online identification need only supply the corrected control derivatives. The bias and the stability derivatives in equation 9 are not required. The problem of applying DI to a system with a nonaffine control mapping has 


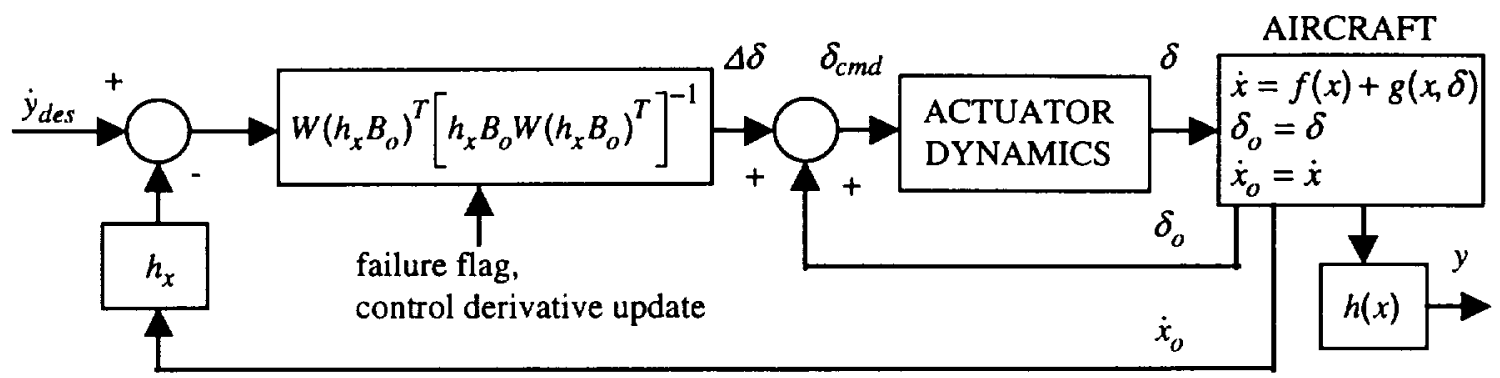

Figure 1. Modified DI Inner-Loop

also been eliminated. Hence, the minimum norm solution for the inverse control is viable. Moreover, once the failure detection and isolation (FDI) algorithm determines which effector has failed, that effector may be easily taken off line and the remaining controls reallocated by simply setting the corresponding weighting to zero. The control does require measurements of $\dot{x}_{o}$ and $\delta_{o}$. In the coming section, $\dot{x}_{o}$ will be constructed from a special set of accelerometer measurements. It is a topic of current interest on how to construct clean signals of $\dot{x}_{o}$ when the accelerometers are subjected to noise, failure, biases, and contamination due to structural flexibility.

\section{Reconfigurable DI Flight Control}

This section includes key equations for assembling a reconfigurable control law using the modified form of dynamic inversion. Specifically, details are given for obtaining $\dot{x}_{o}$ and the control derivatives. Two sets of control variables will be considered.

Using a standard $\mathrm{x}-\mathrm{y}-\mathrm{z}$ body axes coordinate frame, the equations of motion for a flat-earth, rigid body, symmetrical airplane ${ }^{21}$ can be expressed as

$$
\begin{aligned}
& m a_{x, c g}=m\left[\dot{u}+q_{b} w-r_{b} v+g \sin \theta\right]=X \\
& m a_{y, c g}=m\left[\dot{v}+r_{b} u-p_{b} w-g \cos \theta \sin \phi\right]=Y \\
& -m a_{n, c g}=m\left[\dot{w}+p_{b} v-q_{b} u-g \cos \theta \cos \phi\right]=Z \\
& \dot{p}_{b} I_{x x}-\dot{r}_{b} I_{x z}+q_{b} r_{b}\left(I_{z z}-I_{y y}\right)-p_{b} q_{b} I_{x z}=L \\
& \dot{q}_{b} I_{y y}+p_{b} r_{b}\left(I_{x x}-I_{z z}\right)-r_{b}^{2} I_{x z}+p_{b}^{2} I_{x z}=M \\
& \dot{r}_{b} I_{z z}-\dot{p}_{b} I_{x z}+p_{b} q_{b}\left(I_{y y}-I_{x x}\right)+q_{b} r_{b} I_{x z}=N
\end{aligned}
$$

Here $X, Y$, and $Z$ represent the total aerodynamic and thrust forces (lb), $L, M$, and $N$ represent the total aerodynamic and thrust moments (ft-lb); $I_{x x}, I_{y y}$, $I_{z z}$, and $I_{x z}$ are the moments and products of inertia (slug- $\mathrm{ft}^{2}$ ); $m$ is the mass (slugs); $u, v$, and $w$ represent the linear velocities (ft/s); $\dot{u}, \dot{v}$, and $\dot{w}$ are the respective linear accelerations $\left(\mathrm{ft} / \mathrm{s}^{2}\right) ; p_{b}, q_{b}$, and $r_{b}$ represent the roll, pitch, and yaw rates $(\mathrm{rad} / \mathrm{s}) ; \dot{p}_{b}$, $\dot{q}_{b}$, and $\dot{r}_{b}$ are the respective angular accelerations $\left(\mathrm{rad} / \mathrm{s}^{2}\right)$; and $a_{x, c g}, a_{y, c g}$, and $a_{n, c g}$ are the accelerations corresponding to the sum of inertial and gravitational forces as sensed by instrumentation mounted at the cg. The following auxiliary equations must be added to the equations of (18)

$$
\begin{aligned}
& \dot{\theta}=q_{b} \cos \phi-r_{b} \sin \phi \\
& \dot{\phi}=p_{b}+q_{b} \tan \theta \sin \phi+r_{b} \tan \theta \cos \phi .
\end{aligned}
$$

The total forces and moments can generally be decomposed in the form

$$
X=X_{a}+X_{t}+X_{\delta a}
$$

where $X_{a}$ is the component of axial force due to the baseline aerodynamics, $X_{t}$ is the axial force component due to engine thrust which includes the effects of pitch and yaw thrust vectoring, and $X_{\delta a}$ is the axial force due to aerodynamic effector deflection. Let $X_{\tilde{\alpha}}$ denote that portion of $X_{t}$ due to thrust vectoring.

Consulting equations 18 and 19 , let $\dot{x}_{o}$ correspond to the state vector

$$
x=\left[u, w, q_{b}, \theta, v, p_{b}, r_{b}, \phi\right]^{T} .
$$

The linear and angular accelerations will be obtained from a special grouping of accelerometers to be discussed in the next section. $\dot{\theta}$ and $\dot{\phi}$ are obtained from equation 19. Some economy can be realized in constructing $\dot{x}_{o}$ since only those elements multiplying the nonzero columns of $h_{x}$ are needed in the control. Prior to selecting control variables which determine $h_{x}$, the nonlinear control mapping $g(x, \delta)$ used to define the control derivatives is given.

Solving for the linear and angular accelerations in equation 18 , with the decomposition used in equation 20 , and noting the part due exclusively to control yields 


$$
g(x, \delta)=\left[\begin{array}{c}
\left(X_{\delta u}+X_{\delta}\right) / m \\
\left(Z_{\delta u}+Z_{\delta t}\right) / m \\
\left(\left(M_{\delta u}+M_{\delta}\right) / I_{y y} / 180 / \pi\right. \\
0 \\
\left(Y_{\delta a}+Y_{\delta}\right) / m \\
{\left[\begin{array}{cc}
I_{x x} & -I_{x z} \\
-I_{x z} & I_{y y}
\end{array}\right]^{-1}\left\{\begin{array}{c}
L_{\delta a}+L_{\delta x} \\
N_{\delta a}+N_{\delta}
\end{array}\right\} 180 / \pi} \\
0
\end{array}\right] .
$$

In equation 22, all angular accelerations are converted to units of $\mathrm{deg} / \mathrm{s}^{2}$.

In this paper, two sets of control variables are considered for reconfiguration. In one set,

$$
y=h(x)=\left[a_{b}, p_{s}, \beta-0.2 r_{s}\right]^{T}
$$

both the forces and moments of the effectors are manipulated to produce the desired dynamics. Here, $\beta$ represents the sideslip, and $p_{s}$ and $r_{s}$ represent stability axis roll and yaw rates. Force manipulation is required due to the $(u, w)$ dependence of angle of attack, $\alpha$, in $p_{s}$ and $r_{s}$ and the $(u, v, w)$ dependence of $\beta$.

In the second set,

$$
y=h(x)=\left[q_{b}, p_{b}, r_{b}\right]^{T}
$$

only the moments are used to produce the desired dynamics (not the same desired dynamics as the first set). The control resulting from these two sets of control variables will be referred to as the 'Force and Moment' (F\&M), and 'Moment', (M), approaches to reconfiguration, respectively. Details for constructing $h_{x}$ can be found in reference 22 .

\section{Accelerometer Measurements}

Determination of the state acceleration vector $\dot{x}_{o}$ is an important factor in the proposed modified DI control law approach. Potential measurement sources for the elements of this vector include linear accelerometers and numerically differentiated angular rates. Both sources have been chosen to get independent data. Angular acceleration data is derived from a unique implementation of linear accelerometer measurements, in addition to the differentiated rates. This accelerometer implementation could allow a significant amount of sensor redundancy and noise reduction, although these aspects have not been explored at this time. In addition, linear accelerometers are needed for the force and moment (F\&M) control approach that requires state accelerations.

In figure 2 three arbitrary sensor groups $\bar{a}_{i}$ $(i=1,2,3)$ are shown, where each sensor group consist of

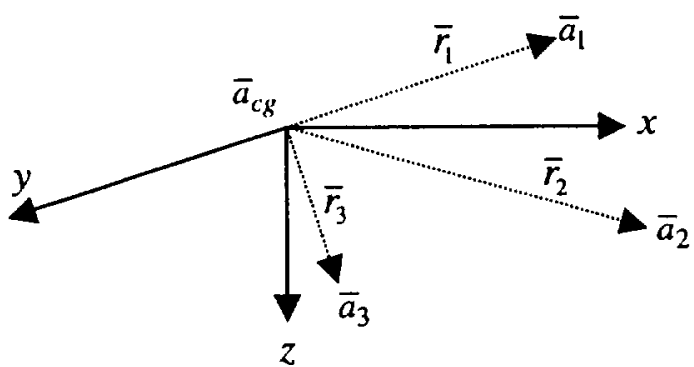

Figure 2. Accelerometer Sensor Group Configuration. three orthogonal linear accelerometers $\left(a_{x i}, a_{y i}, a_{z i}\right)$ located a distance $\bar{r}_{i}$ from the center of gravity (cg). The general accelerometer equation, excluding sensor noise, bias, and airplane flexibility effects, is

$$
\bar{a}_{i}=\bar{a}_{c g}+\dot{\bar{\omega}} \times \bar{r}_{i}+\bar{\omega} \times \bar{\omega} \times \bar{r}_{i}
$$

where $\bar{\omega}$ is a vector of body-axis angular rates $\left(p_{b}, q_{b}, r_{b}\right), \dot{\bar{\omega}}$ is a vector of body-axis angular accelerations $\left(\dot{p}_{b}, \dot{q}_{b}, \dot{r}_{b}\right)$, and $\bar{r}_{i}$ is a position vector of the distances $\left(x_{i}, y_{i}, z_{i}\right)$ to the cg. Body-axis angular accelerations $\left(\dot{p}_{b}, \dot{q}_{b}, \dot{r}_{b}\right)$ are obtained by taking the difference between any two linear accelerometer sensor groups as

$$
\dot{\bar{\omega}} \times \Delta \bar{r}_{i-j}=\left(\bar{a}_{i}-\bar{a}_{j}\right)-\bar{\omega} \times \bar{\omega} \times \Delta \bar{r}_{i-j}
$$

where $\Delta \bar{r}_{i-j}$ represents the distance $\bar{r}_{i}-\bar{r}_{j}$, $\{i, j=1,2,3 ; i \neq j\}$. One important aspect is that the distance between sensor groups, and not the distance to the $\mathrm{cg}$, is needed for the angular acceleration solution. A total of nine equations can be generated from equation 24. In generic form these are

$$
\begin{aligned}
& -\dot{r}_{b} \Delta y_{i-j}+\dot{q}_{b} \Delta z_{i-j}=A_{i-j} \\
& \dot{r}_{b} \Delta x_{i-j}-\dot{p}_{b} \Delta z_{i-j}=B_{i-j} \\
& -\dot{q}_{b} \Delta x_{i-j}+\dot{p}_{b} \Delta y_{i-j}=C_{i-j} \quad i, j=1,2,3 ; i \neq j
\end{aligned}
$$

where the right hand side is

$$
\begin{aligned}
A_{i-j}= & \left(a_{x, i}-a_{x, j}\right)+\left(q_{b}^{2}+r_{b}^{2}\right) \Delta x_{i-j} \\
& -p_{b} q_{b} \Delta y_{i-j}-p_{b} r_{b} \Delta z_{i-j} \\
B_{i-j}= & \left(a_{y, i}-a_{y, j}\right)-p_{b} q_{b} \Delta x_{i-j} \\
& +\left(p_{b}^{2}+r_{b}^{2}\right) \Delta y_{i-j}-q_{b} r_{b} \Delta z_{i-j} \\
C_{i-j}= & -\left(a_{n, i}-a_{n, j}\right)-p_{b} r_{b} \Delta x_{i-j} \\
& -q_{b} r_{b} \Delta y_{i-j}+\left(p_{b}^{2}+q_{b}^{2}\right) \Delta z_{i-j}
\end{aligned}
$$

with the last equation in (28) written in terms of normal acceleration.

There are multiple solutions for $\dot{p}_{b}, \dot{q}_{b}$, and $\dot{r}_{b}$. Only five of the nine accelerometers are required with the stipulation that at least one accelerometer 
measurement must come from each of the three sensor groups. Sensor redundancy has not been explored in this paper, but it appears that many combinations can be used for FDI. In addition, by using multiple solutions the effects of sensor noise and bias may be reduced by some type of averaging technique.

In this paper, $\left(\dot{p}_{b}, \dot{q}_{b}, \dot{r}_{b}\right)$ are derived from a leastsquares solution of 12 equations, 9 from equation (27) and 3 equations from the numerically differentiated rate gyro signals. All equations are weighted equally, but if an FDI system was available equations associated with failed accelerometers could be weighted to zero. It is assumed that the rate gyros would have a separate FDI system.

The next step is to calculate $\bar{a}_{c g}$ using equation 25 , which requires knowledge of $\bar{r}_{i}$. All control designs use models that are based upon some fixed $\mathrm{cg}$ location, so this restriction is not unique. Nine equations are solved, 3 equations each for $a_{x, c g}, a_{y, c g}$, and $a_{n, c g}$.

The 3 equations along each axis are averaged to get the linear accelerations.

State accelerations are required for the $F \& M$ approach and are calculated by rearranging equation 18 as

$$
\begin{aligned}
& \dot{u}=a_{x, c g}-g \sin \theta+v r_{b}-w q_{b} \\
& \dot{v}=a_{y, c g}+g \sin \phi \cos \theta-u r_{b}+w p_{b} \\
& \dot{w}=-a_{n, c g}+g \cos \phi \cos \theta+u q_{b}-v p_{b}
\end{aligned}
$$

with units in $\mathrm{ft} / \mathrm{s}^{2}$. The state acceleration vector $\dot{x}_{0}$ is set up with eight states in the order shown in equation 21.

When the $\mathrm{M}$ control approach is used only three accelerations are required specifically $\left(\dot{q}_{b}, \dot{p}_{b}, \dot{r}_{b}\right)$ and equation 29 is not used. The linear accelerations at the $\mathrm{cg}$ are still used, as discussed in the Flying Qualities section.

\section{Control Derivatives}

For this study, complete tables of control derivatives have been generated from the aerodynamic database. There are at least three reasons for doing this.

1. The modified dynamic inversion control must carry a representation of this control derivative database.

2. Problems with the proposed control redesign methodology are separated from potential problems with real time system identification methodology.

3. Tables provide a baseline for the real time system identification method.
The tables are also useful when various uncertainties and time delays are injected into the control to quantify. worst case scenarios regarding the combined FDI and parameter identification processes. Here, control derivatives are required for both the aerodynamic and thrust vectoring controls. Thrust vectoring control derivatives result analytically from partials taken of functional expressions in reference 22 . Only the aerodynamic control derivatives will be considered in this paper.

In the typical airplane simulation, force and moment aerodynamic data are defined in tables that are generated from wind tunnel and flight data. These tables, which require interpolation, usually contain nondimensional coefficients $\left(c_{i}\right)$ that are nonlinear functions of many airplane variables, for example $\alpha$, $\beta$, Mach, altitude, and $\delta$. Representative of the aerodynamic forces and moments due to controls, consider only the axial force component

$$
X_{\delta a}=c_{\delta a, x} \bar{q} S
$$

where $\bar{q}$ is dynamic pressure $\left(\mathrm{lb} / \mathrm{ft}^{2}\right), S$ is the wing reference area $\left(\mathrm{ft}^{2}\right)$. Aerodynamic force $X_{\delta a}$ has units of lb. The term, $c_{\delta a, x}$, is the nondimensional aerodynamic axial force coefficient due to controls. It should be noted that $c_{\delta a, x}$ represents a sum from several tables including tables of interference coefficients representing the effect of one effector deflecting upstream from another effector. The $c_{\delta a, x}$ terms are also responsible for the nonlinear control mapping. Consulting equation 22 , the first row of $\partial g(x, \delta) / \partial \delta_{a}$ is constructed from the partials $\partial c_{\delta a, x} / \partial \delta_{a}$. In the simulation, the aerodynamic data tables are linearly interpolated. The derivatives, then, are constants between the breakpoints of the tables (actually up to some $\varepsilon$ of the breakpoint). The generated derivative tables have been constructed with this in mind. In testing the on-line control design algorithm, the derivative tables are interpolated along with the original aerodynamic database tables using the simulation's proprietary interpolation scheme.

\section{Flying Oualities}

In this section, equation 3 defining the desired dynamic behavior for the control variables (CV) is considered. Referred to as a 'command model', equation 3 establishes the flying qualities of the closedloop system. The three control variables and their respective command models are considered separately 
since theoretically the inner DI loop has produced a decoupled integrator block relating $\dot{y}_{\text {des }}$ to $y$.

When a slower variable $y_{o l}$ is to be controlled, another outer loop can be wrapped around the inner loops as illustrated in figure 3 . Here the outer loop bandwidth is defined as $\omega_{o l}$. A nonlinear function, $\mathrm{NL}$, is included to relate $\dot{y}_{o l, d e s}$ to $y_{c}$. The reference signal is used in the simulation section for comparison with the desired system response.

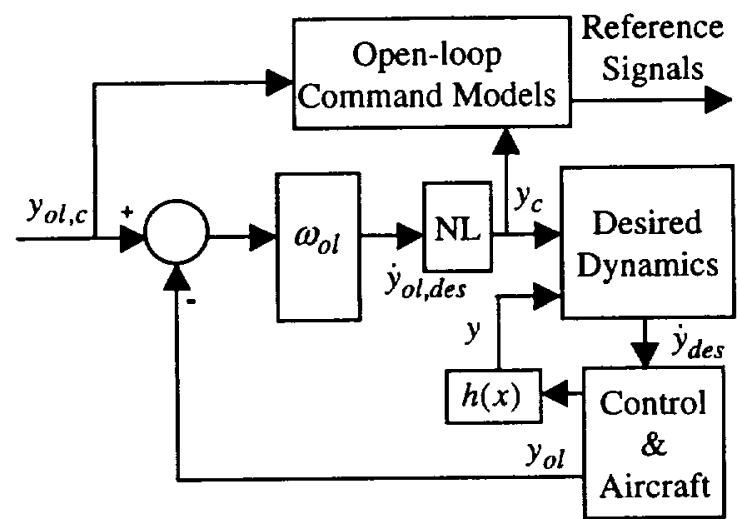

Figure 3. Outer loop configuration

Transfer functions selected for the command models are typically found in the military specifications $^{19}$. Command models in this paper vary with selection of either the force-and moment approach or the moment approach.

\section{Force and Moment (F\&M) Command Models}

The longitudinal, lateral, and directional axes command-models for the F\&M approach are respectively

$$
\begin{aligned}
& \frac{y_{l o n}}{y_{l o n, c}}=\frac{K_{l o n} \omega_{l o n}^{2}\left(s+\omega_{n, l o n}\right)}{s^{2}+2 \zeta_{l o n} \omega_{l o n} s+\omega_{l o n}^{2}} \\
& \frac{y_{l a t}}{y_{l a t, c}}=\frac{\omega_{l a t}}{s+\omega_{l a t}} \\
& \frac{y_{d i r}}{y_{d i r, c}}=\frac{\omega_{d i r}^{2}}{s^{2}+2 \zeta_{d i r} \omega_{d i r} s+\omega_{d i r}^{2}} .
\end{aligned}
$$

Simulations for the F\&M approach illustrated in this paper include a pitch-rate command $\left(q_{c}\right)$ for the longitudinal axis, a stability-axis roll-rate command $\left(p_{s, c}\right)$ for the lateral axis, and a linear combination of stability-axis yaw-rate $\left(r_{s, c}\right)$ and sideslip-angle $\left(\beta_{c}\right)$ commands for the directional axis. The lateral and directional axis command models illustrate the mixing of linear and angular variables.
In the lateral axis a second outer loop (as illustrated in figure 3 ) is wrapped around the first outer loop for $p_{s, c}$ to control bank angle $\phi$ during batch simulation runs. The two outer loops together create a combined transfer function that has the form of equation 33. With the second outer-loop frequency defined as $\omega_{p h i}$, the

following relationships can be derived as

$$
\begin{aligned}
& \omega_{\text {lat }}=2 \zeta_{\text {equivalent }} \omega_{\text {equivalent }} \\
& \omega_{\text {phi }}=\frac{\omega_{\text {equivalent }}}{2 \zeta_{\text {equivalent }}}
\end{aligned}
$$

where $\omega_{\text {equivalent }}$ and $\zeta_{\text {equivalent }}$ represent the natural frequency and damping ratio of the combined $2^{\text {nd }}$-order transfer function. These equations are also used in the moment approach that follows. Parameter $\omega_{p h i}$ is the outer-loop bandwidth for approximating the desired bank-angle rate $\dot{\phi}_{\text {des }}$ and is defined as

$$
\dot{\phi}_{\text {des }}=\omega_{p h i}\left(\phi_{c}-\phi\right) \text {. }
$$

A nonlinear equation is inserted into the control loop to approximately relate $\dot{\phi}_{\text {des }}$ to $p_{s, c}$ as

$p_{s, c} \cong\left(\dot{\phi}_{\text {des }}-\left(q_{b} \sin \phi+r_{b} \cos \phi\right) \tan \theta\right) \cos \alpha$

Simulation results will show that these equations give a very close approximation to the idealized second-order open-loop reference response using $\omega_{\text {equivalent }}$ and

$\zeta_{\text {equivalent }}$.

\section{Moment (M) Command Models}

The moment approach only uses angular bodyrates for the DI loop and only three angular control derivatives are required. First-order command models are used for $p_{b}, q_{b}$ and $r_{b}$ with transfer functions similar to equation 32. A second outer-loop similar to the one discussed for the lateral axis above is used for all of the control loops. The only difference in the lateral loop is the nonlinear equation for body-axis roll rate command

$$
p_{b, c}=\dot{\phi}_{d e s}-\left(q_{b} \sin \phi+r_{b} \cos \phi\right) \tan \theta \text {. }
$$

The longitudinal $\mathrm{CV}$ is $\alpha$ and the directional $\mathrm{CV}$ is $\beta$. Parameters $\omega_{\alpha}$ and $\omega_{\beta}$ are the outer-loop bandwidth frequencies for approximating $\dot{\alpha}_{d e s}$ and $\dot{\beta}_{\text {des }}$ respectively as

$$
\begin{aligned}
& \dot{\alpha}_{\text {des }}=\omega_{\alpha}\left(\alpha_{c}-\alpha\right) \\
& \dot{\beta}_{\text {des }}=\omega_{\beta}\left(\beta_{c}-\beta\right) .
\end{aligned}
$$

The nonlinear expressions between $\dot{\alpha}_{d e s}$ and $q_{b, c}$ and between $\dot{\beta}_{d e s}$ and $r_{b, c}$ are 


$$
\begin{aligned}
q_{b, c} \approx & \dot{\alpha}_{d e s}+\frac{g\left(a_{n, c g}-\cos \theta \cos \phi\right)}{V \cos \alpha \cos \beta} \\
& -\tan \alpha \tan \beta \dot{\beta}_{d e s}+\frac{p_{b, c} \tan \beta}{\cos \alpha} \\
r_{b, c} \approx & -\frac{\dot{\beta}_{d e s}}{\cos \alpha}+\frac{g\left(a_{y, c g}+\cos \theta \sin \phi\right)}{V \cos \alpha \cos \beta}+p_{b, c} \tan \alpha .
\end{aligned}
$$

In the next section, examples illustrate the proposed online control design in various failure scenarios for both sets of control variables. Later, an experimental simulation tool is used to determine the stability robustness of the proposed nonlinear closedloop systems.

\section{Simulation Results}

\section{Aircraft Model}

A nonlinear simulation using ATLAS* (Aircraft Trim, Linearization, And Simulation) that includes a highly maneuverable tailless fighter aircraft model with innovative control effectors ${ }^{15,16}$ has been used for this research. Some of the advantages of a tailless aircraft are reduced radar signature and reduced weight and drag. Analysis of the linearized aircraft model shows that it is unstable in the lateral-directional axes at high speed. Table 1 shows the longitudinal and lateral directional eigenvalues (unstable eigenvalues italicized) for three Mach numbers at $25000 \mathrm{ft}$.

Table 1. Eigenvalues of Linearized Airplane Model

\begin{tabular}{|l|l|l|}
\hline Mach & Longitudinal & Lateral-Directional \\
\hline 0.4 & $-0.000812 \pm \mathrm{j} 0.0894$ & $-0.05113 \pm \mathrm{j} 0.1609$ \\
\cline { 2 - 3 } & $-0.5305 \pm \mathrm{j} 1.667$ & $-0.2176 \pm \mathrm{j} 0.7351$ \\
\hline 0.7 & $-0.00328 \pm \mathrm{j} 0.04584$ & 0.02688 \\
\cline { 2 - 3 } & $-0.6621 \pm \mathrm{j} 1.783$ & 0.8625 \\
\cline { 2 - 3 } & & $-0.9073 \pm \mathrm{j} 0.4728$ \\
\hline \multirow{3}{*}{0.9} & 0.1017 & 0.009477 \\
\cline { 2 - 3 } & -0.1140 & 1.446 \\
\cline { 2 - 3 } & $-0.9452 \pm \mathrm{j} 3.556$ & $-1.303 \pm \mathrm{j} 0.3415$ \\
\hline
\end{tabular}

Eleven control effectors are used in this research, but four of these controls are unilateral (operate only in one angular direction) leaving nine effective controls. These effective controls are: 1) left elevon (LE), 2) right elevon (RE), 3) symmetric pitch flap (SPF), 4) all-moving tip (AMT), 5) spoiler-slot-deflector (SSD), 6) left outboard leading edge flap (LOLEF), 7) right outboard leading edge flap (ROLEF), 8) pitch vectoring (PV), and 9) yaw vectoring (YV). The AMT and the SSD controls are unilateral with control

\footnotetext{
- Property of the Flight Controls Branch of Lockheed Martin Tactical Aircraft Systems.
}

effectors on both the left side (LAMT and LSSD) and right side (RAMT and RSSD) of the airplane.

Second-order actuator dynamics are included in al! of the simulation runs. These dynamics are composed of two real poles with transfer function

$$
T_{a}=\frac{\omega_{a 1}}{s+\omega_{a 1}} \frac{\omega_{a 2}}{s+\omega_{a 2}}
$$

where the pole values are shown in table 2 .

Table 2. Actuator Dynamics

\begin{tabular}{|c|c|}
\hline Control Effector & $\begin{array}{c}\text { Poles } \\
(\mathrm{rad} / \mathrm{s})\end{array}$ \\
\hline LE, RE & 40,100 \\
\hline SPF & 40,100 \\
\hline LAMT, RAMT & 40,100 \\
\hline LSSD, RSSD & 40,100 \\
\hline LOLEF, ROLEF & 18,100 \\
\hline PV & 37,41 \\
\hline YV & 37,41 \\
\hline
\end{tabular}

\section{Force and Moment (F\&M) Approach}

The first DI control implementation uses a 6-DOF force and moment ( $F \& M$ ) approach similar to that used in the achievable dynamics research ${ }^{22}$, and discussed in a previous section of this paper. Three control variables (CV) are defined as shown in equation (23). In this vector the longitudinal $\mathrm{CV}$ is pitch rate, the lateral $\mathrm{CV}$ is stability axis roll rate, and the directional $\mathrm{CV}$ is a linear combination of sideslip and stability axis yaw rate. In the lateral axis, a second outer loop is wrapped around the first outer loop for $p_{s}$ to control bank angle $\phi$ during batch simulation runs.

All command models, corresponding equations and variables are discussed in the section on Flying Qualities. Values for the command model parameters are shown in table 3 with units of $\mathrm{rad} / \mathrm{s}$ used for all frequencies.

Table 3. Command Model Parameters, F\&M Approach

\begin{tabular}{|c|c|c|c|}
\hline Parameter & Value & Parameter & Value \\
\hline$\varsigma_{\text {lon }}$ & 0.7 & $\omega_{\text {lat }}$ & 2 \\
\hline$\omega_{\text {lon }}$ & 5 & $\varsigma_{\text {dir }}$ & 0.7 \\
\hline$\omega_{n, l o n}$ & 3 & $\omega_{\text {dir }}$ & 3 \\
\hline$K_{\text {lon }}$ & $1 / 3$ & $\omega_{\text {phi }}$ & 0.75 \\
\hline
\end{tabular}

In all of the examples shown below, the controller sampling-frequency is $100 \mathrm{hz}$ and the control effectiveness matrix is updated from tables every iteration. The nonlinear simulation includes the actuator dynamics described above, hinge moments, rate limits, and position limits. As discussed earlier in this paper, a 
linear stability matrix $A_{o}$ is not required for this DI approach.

For the baseline example, the airplane was trimmed straight and level at 25000 feet and Mach 0.7. A directional channel doublet of amplitude 5 degrees was commanded during the first 5 seconds. At 7.5 seconds into the simulation, a 50 degrees bank angle step was commanded, and between 11 and 16 seconds a $5 \mathrm{deg} / \mathrm{sec}$ pitch doublet was commanded. In addition, at 0.05 second into the simulation, the throttle was increased $60^{\circ}$ above than the trim value. This large increase in throttle along with the downward direction of the velocity vector caused Mach number to increase from 0.7 to near 0.9 over the 20 -second simulation.

Results are illustrated in the four plots shown in figure 4. Solid lines represent the actual control variables, long-dashed lines represent input commands, and dotted lines represent reference signals from openloop command models. The top plot for the longitudinal doublet command shows that the $q_{b}$ response almost perfectly duplicates the reference signal, except for a little ripple during the $\beta$ movements. The second plot is for the $\phi$ response which also closely overlays the reference signal. The generated error signal is the command $p_{s, c}$ that drives the $p_{s}$ response shown in the third plot. The slight variation between the $p_{s}$ response and its reference signal is probably caused by the nonlinear function relating $\dot{\phi}_{\text {des }}$ and $p_{s, c}$. Finally, the directional doublet command, reference signal, and actual response are shown in the fourth plot. Tracking results are almost as good as the results obtained using the achievable dynamics tool ${ }^{22}$ that did not include actuator dynamics.

Figure 5 has the same set of plots for an example illustrating a change in aerodynamics resulting from two missing surfaces. One second into the simulation both the left elevon and the left SSD are declared missing, which could happen if part of the left wing is damaged. The control system instantaneously knows about the missing surfaces and reconfigures by excluding these controls. Since the SSD controls are unilateral the right SSD is also excluded to prevent the control allocator from commanding the missing control. Recall that a stability matrix isn't required since the changing forces and moments resulting from the revised aerodynamics are completely measured by accelerometers. Comparing these results with the baseline case in figure 4 shows that tracking is only slightly degraded. The system response looks good because there is enough control power to compensate for the failures.
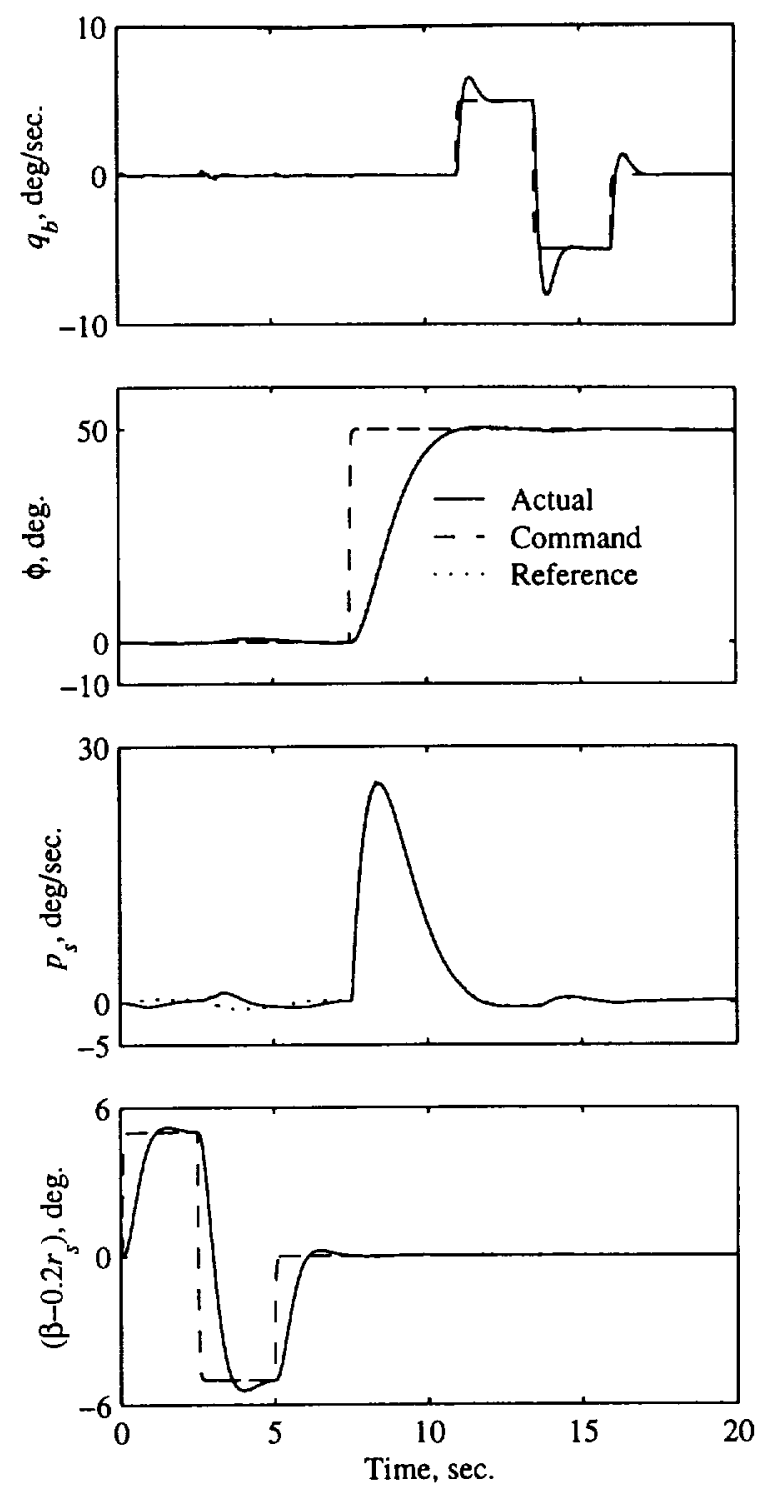

Figure 4.Baseline force and moment example.

The ATLAS aerodynamic database incorporates changes resulting from missing surfaces. Examination of a linear model for this failure condition shows a coupled longitudinal and lateral-directional stability matrix. Eigenvalues for the double missing surface case at Mach 0.7 are shown in table 4. Although the longitudinal and lateral-directional eigenvalues cannot be isolated, they can be compared with those for the Mach 0.7 case in table 1. It appears that the short period has changed significantly from $-0.6621 \pm \mathrm{j} 1.783$ in table 1 to $-0.8151 \pm \mathrm{j} 1.096$ in table 4 . The dutch-roll $(-.9073 \pm j 0.4728$ in table 1$)$ has a smaller change which is $-0.7608 \pm j 0.3934$ in table 4 . The eigenvalue associated with the roll mode $(0.8625$ in table 1$)$ is reduced slightly to 0.7975 in table 4 .

The next F\&M example illustrates results for a double control-effector failure case. At 0.25 second the 
Table 4. Eigenvalues with Missing Surfaces

\begin{tabular}{|c|}
\hline 0.02705 \\
\hline $0.001806 \pm j 0.1141$ \\
\hline 0.7975 \\
\hline$-0.7608 \pm \mathrm{j} 0.3934$ \\
\hline$-0.8151 \pm \mathrm{j} 1.096$ \\
\hline
\end{tabular}
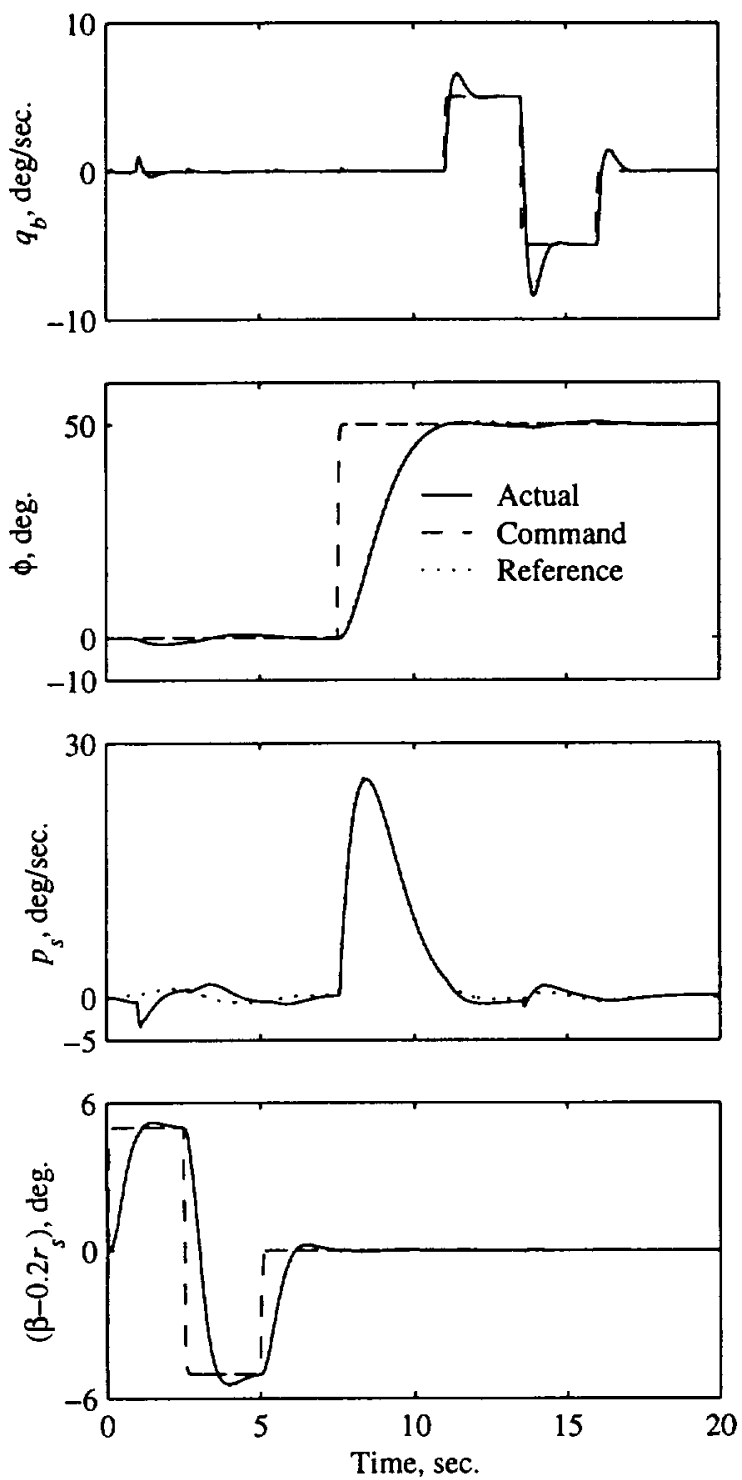

Figure 5. Example of system response to an aerodynamic change due to missing LE and LSSD surfaces using a force and moment approach.

yaw nozzle goes to a stuck maximum position of $15^{\circ}$ left, and at one second the left AMT goes to a stuck maximum position of $60^{\circ}$. Results from two simulation cases are included in figure 6 . One example is for the case where the control system instantly knows about the failures and reconfigures (solid lines) while the second example is for the case where the control system does not know about the failures (dotted lines). The
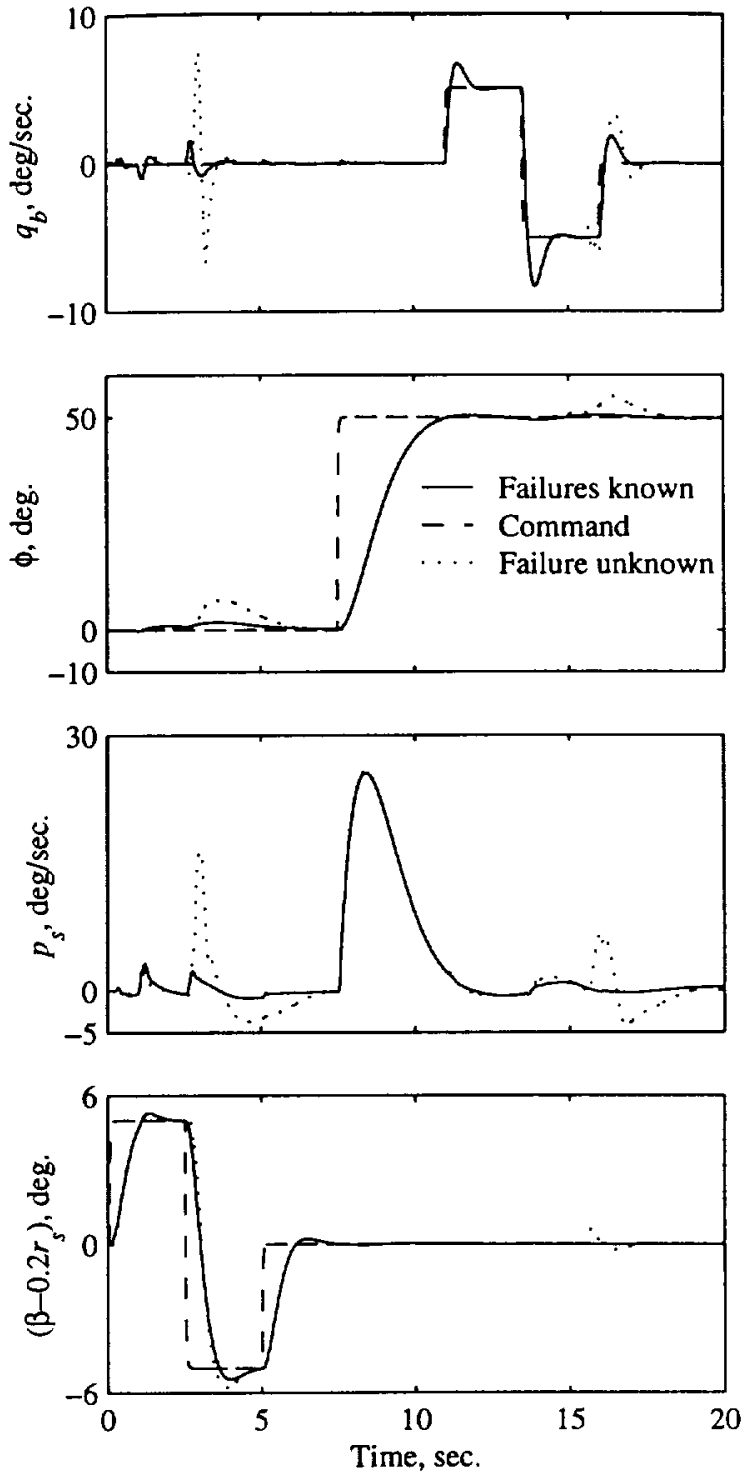

Figure 6. Double failure case example using a force and moment approach.

command is illustrated by long dashes but the reference signal is not included in this figure to avoid clutter. The reconfigured example is clearly superior although both cases successfully complete the simulation run. The non-reconfigured example does a decent tracking job, illustrating robustness to these failures. In the reconfigured example the right $\mathrm{AMT}$ is used to help counteract moments produced by the failed left AMT. In both cases, the remaining control effectors (not shown) go to new positions to compensate for the forces and moments generated by the stuck AMT and the missing thrust moment from the yaw nozzle.

However, in the non-reconfigurable example the left tail is saturated for a short period during the simulation causing the errors seen in the figure. 


\section{Moment (M) Approach}

As discussed in the Flying Qualities section, the main change for the moment approach is that control variables are the three angular rates $\left(p_{b}, q_{b}, r_{b}\right)$ and commands to the DI loop are their respective angular accelerations ( $\dot{p}_{b, \text { des }}, \dot{q}_{b \text {,des }}$, and $\dot{r}_{b, \text { des }}$ ). First-order command models are used for the rates with bandwidths $\omega_{\text {lat }}, \omega_{\text {lon }}$, and $\omega_{\text {dir }}$ respectively. An outer loop is wrapped around each of the inner loops to control slower variables $\alpha, \beta$, and $\phi$ with frequencies $\omega_{\alpha}, \omega_{\beta}$, and $\omega_{p h i}$.

Table 5 contains the parameter values in the following simulations. Variables $\omega_{\text {equivalent }}$ and $\zeta_{\text {equivalent }}$ are defined in equations 34 and 35.

Table 5. Command Model Parameters, M Approach

\begin{tabular}{|c|c|c|c|}
\hline Parameter & Value & $\omega_{\text {equivalent }}$ & $\zeta_{\text {equivalent }}$ \\
\hline$\omega_{\text {lat }}$ & 2 & 1.22 & 0.82 \\
\hline$\omega_{\text {phi }}$ & 0.75 & & \\
\hline$\omega_{\text {lon }}$ & 4.48 & 2.8 & 0.8 \\
\hline$\omega_{\alpha}$ & 1.75 & & \\
\hline$\omega_{\text {dir }}$ & 3.6 & 1.8 & 1.0 \\
\cline { 1 - 2 }$\omega_{\beta}$ & 0.9 & & \\
\hline
\end{tabular}

Figure 7 shows comparison between a baseline simulation and a simulation with three known failures. Since slower variables are being controlled in this example, longer step responses are used as opposed to doublets. In these simulation runs, $\beta$ is commanded to $5^{\circ}$ during the first 5 seconds and then back to null. At 7.5 seconds into the simulation, a $50^{\circ}$ bank-angle step is commanded, and between 11 and 16 seconds a $5^{\circ}$ $\alpha$-step is commanded.

The top three plots show the commands (long dashed line) and responses (solid line for baseline and dashed line for failure case). The remaining 9 plots represent control signals as measured at the actuator output. Only the leading edge outboard flaps are not shown since they are insignificant. Both baseline and failure case results are included for each plot.

Three different failures are demonstrated: 1) at 0.25 second the yaw nozzle fails at $0^{\circ}, 2$ ) at 1 second the left elevon is missing, and 3 ) at 1 second the left AMT goes to maximum deflection of $60^{\circ}$. The missing elevon and stuck AMT cause aerodynamic changes. One main conclusion from these results is that the reconfigured control system behaves almost as good as the original control system. However, the plots illustrate that large changes in actuator control positions are required to accommodate the three failures.

\section{Robustness Evaluation}

Stability and robustness evaluation was done experimentally using a simulation tool named RASCLE $^{23}$ (Robustness Analysis for Simulation-based Control Law Evaluation) that was recently developed under contract to NASA Langley Research Center. Basically, RASCLE inserts selected uncertainties (gains, delays, bias, noise) into the nonlinear simulation and performs a multitude of simulation runs to determine the uncertainty values that cause the control system to become unstable. In particular, RASCLE finds the stability boundary point (or surface for several simultaneous uncertainties) within a predefined tolerance. For each uncertainty combination the nonlinear simulation is first excited by input commands (doublets in this paper), and then selected signals are monitored for stability. RASCLE performs a power spectral density analysis on the monitored data after each simulation to determine stability and the dominant frequency for each monitored signal. One major advantage of using this type of analysis is that nonlinear-variables and functions are included.

RASCLE assumes a stable system with a nominal gain uncertainty equal to one and a time delay of zero. Gain uncertainties are separated into two parts, gains greater than nominal and gains less than nominal. Time delays can also be converted to phase angle by using the dominant frequency that has been determined.

For this paper, gain and delay uncertainties were inserted separately at several locations to find the boundary points. Referencing notation in figure 1, uncertainties were inserted at: 1) $y$ and other state measurements, 2) accelerometer measurements for $\dot{x}_{o}$, 3) actuator control commands $\left.\delta_{c m d}, 4\right)$ actuator position measurements $\delta_{o}$, and 5) control derivatives $B_{o}$ where all derivatives for any control are treated together. Specifically, the following measurements have been made for both the F\&M and the $M$ approaches:

1. $\quad y$ and other state measurements: body rates

$\left(p_{b}, q_{b}, r_{b}\right)$, linear velocities $(\mathrm{u}, \mathrm{v}, \mathrm{w})$, angles $(\alpha, \beta)$

2. Accelerometer measurements for $\dot{x}_{o}$ (see definitions in the Accelerometer Measurements section): $a_{x, 1}, a_{y, 1}, a_{n, 1}, a_{x, 2}, a_{y, 2}, a_{n, 2}, a_{x, 3}$, $a_{y, 3}, a_{n, 3}$ 

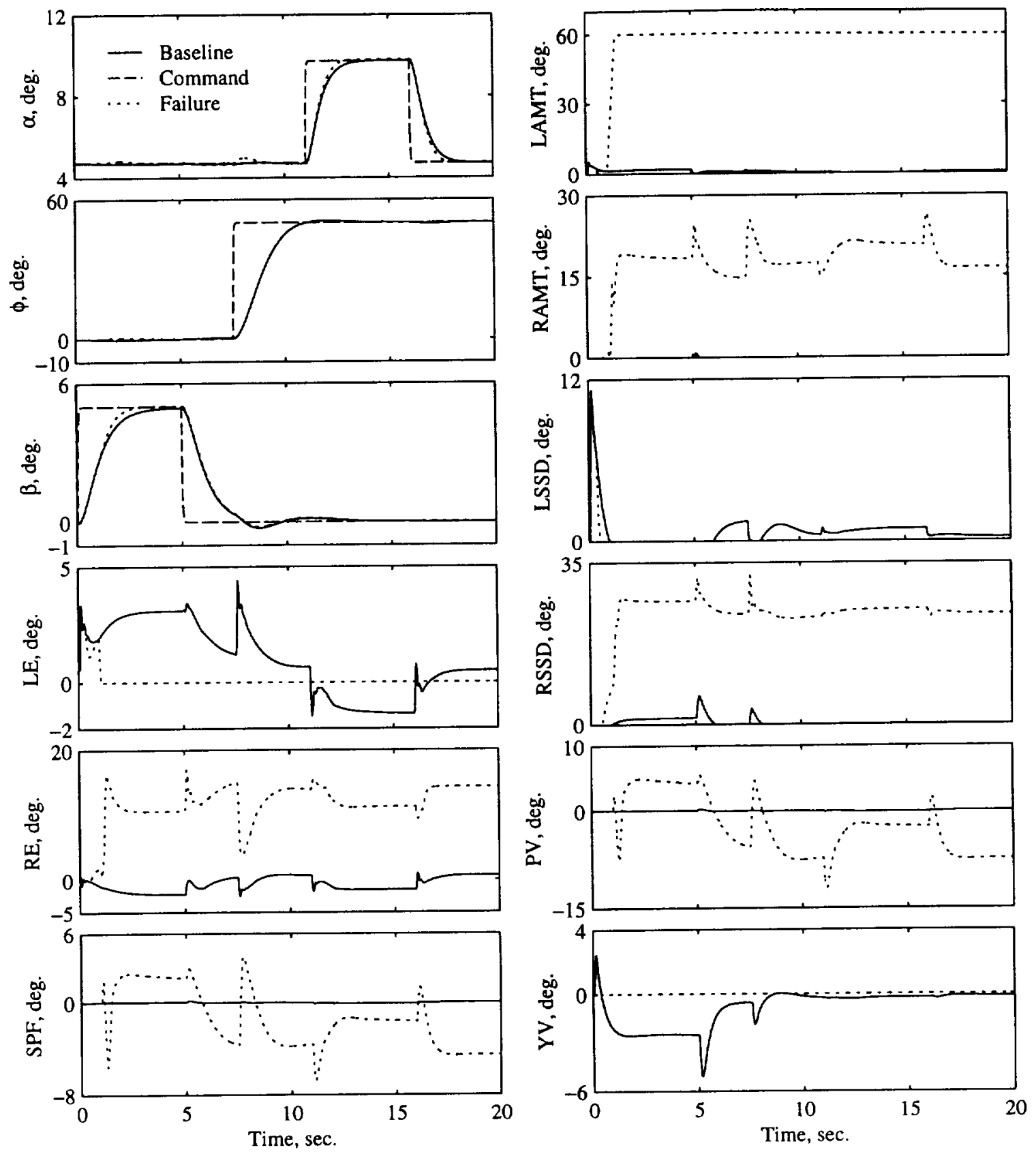

Figure 7. Moment-control showing baseline and 3-failure example.

3. Actuator Control commands $\delta_{\text {cmd }}$ (see definitions in the Simulation Results section): LE, RE, SPF, LAMT, RAMT, LSSD, RSSD, LOLEF, ROLEF, $\mathrm{PV}, \mathrm{YV}$

4. Actuator position measurements $\delta_{o}$ (same 11 actuator definitions as above but different points)
5. Control derivatives $B_{0}$ : using columns for derivatives associated with actuators LE, RE, SPF, AMT, SSD, LOLEF, ROLEF, PV, and YV. 
Table 6. Uncertainty Results

\begin{tabular}{|c|c|c|c|c|c|c|c|c|}
\hline \multirow[b]{2}{*}{ Variable } & \multicolumn{4}{|c|}{ Force and Moment (F\&M) Approach } & \multicolumn{4}{|c|}{ Moment (M) Approach } \\
\hline & Gain $\geq$ & Gain $<$ & Delay & Phase & Gain $>$ & Gain $<$ & Delay & Phase \\
\hline & $\mathrm{db}$ & $\mathrm{db}$ & $\sec$ & deg & $\mathrm{db}$ & $\mathrm{db}$ & $\mathrm{sec}$ & $\operatorname{deg}$ \\
\hline \multicolumn{9}{|l|}{ state variables } \\
\hline$p_{b}$ & $>20$ & $<-20$ & $>2$ & N.A. & $>20$ & $<-20$ & 0.6094 & 86 \\
\hline$q_{b}$ & 12.7 & $<-20$ & 0.1328 & 58 & 17 & $<-20$ & 0.2969 & 89 \\
\hline$r_{b}$ & $>20$ & $<-20$ & 0.2656 & 121 & $>20$ & $<-20$ & 0.625 & 88 \\
\hline \multicolumn{9}{|l|}{$\frac{b}{\alpha}$} \\
\hline$\beta$ & $>20$ & $<-20$ & 0.3281 & 58 & $>20$ & $<-20$ & 1.5 & 79 \\
\hline \multicolumn{9}{|l|}{ accelerometers } \\
\hline$a_{n, 1}$ & $>20$ & $<-20$ & 0.3906 & 76 & $>20$ & -5.2 & 0.2344 & 29 \\
\hline$a_{n, 2}$ & 8.1 & $<-20$ & 0.2281 & 108 & 2.6 & $<-20$ & 0.1094 & 78 \\
\hline$a_{n, 3}$ & 7.4 & $<-20$ & 0.0797 & 73 & 3.5 & $<-20$ & 0.1562 & 85 \\
\hline \multicolumn{9}{|c|}{ actuator control commands } \\
\hline LE & 10.2 & $<-20$ & 0.4219 & 163 & 12 & $<-20$ & 0.6875 & 169 \\
\hline RE & 12 & $<-20$ & 0.4219 & 163 & $>20$ & $<-20$ & $>2$ & N.A. \\
\hline LAMT & 19.1 & $<-20$ & $>2$ & N.A. & 18.4 & $<-20$ & $<-20$ & $>2$ \\
\hline RAMT & $>20$ & $<-20$ & $>2$ & N.A. & 17.1 & $<-20$ & $<-20$ & $>2$ \\
\hline \multicolumn{9}{|c|}{ control derivative columns } \\
\hline YV & 14.7 & $<-20$ & $>2$ & N.A. & $>20$ & $<-20$ & $>2$ & N.A. \\
\hline
\end{tabular}

Gain uncertainty boundary limits were set at a factor of $10( \pm 20 \mathrm{db})$ and the time delay limit was set at 2 seconds. Uncertainty results are shown in table 6. Only those variables with at least one uncertainty within the boundary limit for which the system is unstable are shown in the table. The rest of the variables have large uncertainty limits. Phase angle has been calculated where applicable; time delays greater than the bound do not have a boundary frequency and are not applicable (N.A.). Tolerance for the phase angle calculations is $\pm 9^{\circ}$ based upon the number of samples taken for each simulation. All data is for an initial trim condition of Mach 0.7 at 25000 feet. It appears that the only variables that do not meet military specification $\left(6 \mathrm{db}\right.$ and $\left.45^{\circ}\right)$ are the normal accelerometers using the $M$ approach. All of the normal accelerometers have at least one low gain margin and $a_{n, 1}$ has a low phase margin. A probable reason why the $F \& M$ approach appears more robust for the normal accelerometer uncertainties is that the longitudinal DI controller uses a pitch rate command whereas in the $M$ approach an $\alpha$-command is used in an outer loop to drive the DI pitch rate command. Normal acceleration is used in the nonlinear gain, as described in the Flying Qualities section. These results indicate a need to integrate a good accelerometer FDI (failure detection and isolation) system.
RASCLE initially found poor margins in the actuator control commands for TE and RE. The main reason is that the actuator loop is effectively an integrator created by feeding back the actuator position $\delta_{o}$ (see figure 1). Uncertainties in this loop are accumulated and could drive a control into saturation.

This problem was reduced by adding a simple firstorder actuator reference model driven by $\delta_{c m d}$.

Outputs from the reference model are compared with corresponding outputs from the real actuator and the signal with the lowest absolute value is selected. When the real actuator outputs are used, the reference model states are updated to the actual states. A reference model is used for all actuator signals.

\section{Conclusion}

A modified version of nonlinear dynamic inversion provides the underlying framework of the proposed online design control law. The proposed control is less dependent on the onboard model than other dynamic inversion based methods in that it only requires information on control derivatives. No stability derivatives are required in the implementation. In failure, aerodynamic changes cause force and moment changes on the aircraft. The force and moment changes can be measured by the accelerometers. The proposed 
control exploits this result to regain control of the damaged vehicle. Sensitivity to the onboard model is reduced in favor of obtaining a more robust measure of the vehicle's accelerations.

Implementation issues associated with obtaining the vehicle's linear and angular accelerations have been addressed. Angular acceleration data is derived from a unique implementation of linear accelerometer measurements, in addition to differentiated rate signals, using a least squares solution. Based on the angular acceleration, the measured linear accelerations are translated back to the $\mathrm{cg}$ and averaged. The accelerometer implementation used could allow a significant amount of sensor redundancy and noise reduction, although these aspects have not been explored.

In an attempt to further promote robustness, two control structures involving two sets of control variables were investigated. The first, the force and moment (F\&M) approach requires both linear and angular control derivatives. The second, moment (M) approach requires only the angular control derivatives. The derivatives required are determined by the control variables specified in the inner dynamic inversion loop. The F\&M control uses a mixture of force type control variables $(\alpha$ and $\beta$ ) and moment type control variables $\left(p_{b}, q_{b}, r_{b}\right)$. The $\mathrm{M}$ control uses only moment type variables in the inner dynamic inversion loop. Command models containing flying qualities were developed for both approaches, with $\mathrm{M}$ control requiring a more elaborate outer-loop structure involving force type control variables to obtain handling comparable to the F\&M approach.

Simulation results indicate both methods are capable of online control design subject to multiple failures during complex maneuvering. The baseline simulation results illustrate excellent tracking for the $F \& M$ and $M$ control approaches. The F\&M performance is slightly better than the $M$ performance. However, the F\&M approach utilizes more involved control variables and more control derivatives in its inner loop. The $\mathrm{M}$ approach utilizes non-linear expressions to relate the outer-loop force variables to the DI moment variables. Both controls have been shown to accommodate aerodynamic changes without the use of stability derivative information. The system response looks good when there is enough control power to compensate for the failures. An F\&M example illustrated robustness to some failures when the failure information was unknown.

An experimental simulation tool (RASCLE) was used to evaluate the control system's stability robustness. Gain and phase uncertainties were inserted at five major control system locations and 48 variables were evaluated. Only the normal accelerometer uncertainties resulted in the control not meeting the desired gain margin $(6 \mathrm{~dB})$ and phase margin $\left(45^{\circ}\right)$ for the uncertainty used. This analysis indicates the need for a good FDI system to eliminate defective accelerations.

\section{Acknowledgement}

The nonlinear simulation tool ATLAS used in this research is the property of the Flight Controls Branch of Lockheed Martin Tactical Aircraft Systems.

\section{References}

${ }^{1}$ Honeywell Technology Center; Lockheed Martin Skunk Works and Lockheed Martin Tactical Aircraft Systems: Application of Multivariable Control Theory to Aircraft Control Laws. WL-TR-96-3099, Final Report for March 1993 to March 1996, May 1996.

${ }^{2}$ Monaco, J., Ward, D., and Bird, R.:

Implementation and Flight Test Assessment of an Adaptive, Reconfigurable Flight Control System. Proc. AIAA Guidance, Navigation, and Control Conference, New Orleans, LA, AIAA-97-3738, Aug. 1997.

${ }^{3}$ Ward, D., Monaco, J., Barron, R., Bird, R., Virnig, J., and Landers, T.: Self-Designing Controller: Design, Simulation, and Flight Test Evaluation. SBIR Phase II Final Technical Report, Contract F49620-94-C-0087, Barron Associates, Inc., Nov. 13, 1996.

${ }^{4}$ Eberhardt, R. and Ward, D.: Indirect Adaptive Flight Control System Interactions. Int. J. Robust Nonlinear Control, Vol. 9, pp. 1013-1031, 1999.

${ }^{5}$ Corvin, J., Havern, W., Hoy, S., Norat, K., Urnes, J., and Wells, E.: Self-Repairing Flight Control System, Vol. I: Flight Test Evaluation on an F-15 Aircraft. WL-TR-91-3025, Final Report for Oct. 1987-Dec. 1990, Aug. 1991.

${ }^{6}$ The Boeing Company: Intelligent Flight Control Advanced Concept Program. BOEING-STL 99P0040, Final Report, May 15, 1999.

${ }^{7}$ Halyo, N., Direskeneli, H., and Taylor, D.: A Stochastic Optimal Feedforward and Feedback Control Methodology for Superagility. NASA CR-4471, Nov. 1992.

${ }^{8}$ Ostroff, A.: Study of a Simulation Tool to Determine Achievable Control Dynamics and Control Power Requirements with Perfect Tracking. NASA TM-1998-208699, Aug. 1998.

${ }^{9}$ Brinker, J., and Wise, K.: Reconfigurable Flight Control for a Tailess Advanced Fighter Aircraft. Proc. AIAA Guidance, Navigation, and Control Conference, Boston, MA , AIAA-98-4107, pp. 75-87, Aug. 1998. 
11"Kim, B., and Calise, A.: Nonlinear Flight Control Using Neural Networks, J. Guidance, Control, and Dynamics, Vol. 20, No. 1, Jan.-Feb. 1997.

"McFarland, M.: Augmentation of GainScheduled Missile Autopilots using Adaptive Neural Networks. Proc. AIAA Guidance, Navigation, and Control Conference, Boston, MA, AIAA-98-4491, pp. 1786-1792, Aug. 1998.

${ }^{12}$ Calise, A., Sharma, M., and Corban, J.: An Adaptive Autopilot Design for Guided Munitions. Proc. AIAA Guidance, Navigation, and Control Conference, Boston, MA, AIAA-98-4490, pp. 1776-1785, Aug. 1998.

${ }^{13}$ Calise, A., Lee, S., and Sharma, M.: Direct Adaptive Reconfigurable Control of a Tailless Fighter Aircraft. Proc. AIAA Guidance, Navigation, and Control Conference, Boston, MA, AIAA-98-4108, pp. 88-97, Aug. 1998.

${ }^{14}$ Smith, P.: A Simplified Approach to Nonlinear Dynamic Inversion Based Fight Control. AIAA Atmospheric Flight Mechanics Conference, AIAA-984461, pp. 762-767, August 1998.

${ }^{15}$ Dorsett, K.; and Mehl, D.: Innovative Control Effectors (ICE). WL-TR-96-3043, January 1996.

${ }^{16}$ Dorsett, Kenneth M.; and Fears, Scott P..: Innovative Control Effectors (ICE) Phase II. WL-TR97-3059, August 1997.

${ }^{17}$ Bugajski, D., Enns, D., and Elgersma, M.: A Dynamic Inversion based Control Law with Application to High Angles of Attack Research Vehicle, Proc. AIAA Guidance, Navigation, and Control Conference, 90-3407-CP, pp. 826-836, August 1990.

${ }^{18}$ Buffington, J.M.: Modular Control Law Design for the Innovative Control Effectors (ICE) Tailess Fighter Aircraft Configuration 101-3. AFRL-VA-WPTR-1999-3057, Final Report for 05/01/97-05/01/99, June 1999.

${ }^{19}$ Military Standard Flying Qualities of Piloted Aircraft. MIL-STD-1797A, Jan. 30, 1990.

${ }^{20}$ Morelli, E.A.: Real-Time Parameter Estimation in the Frequency Domain. AIAA Guidance, Navigation, and Control Conference, AIAA 99-4043, August 9-11, 1999.

${ }^{21}$ Roskam, J.: Airplane Flight Dynamics and Automatic Flight Controls, Part I. Published by Roskam Aviation and Engineering Corporation, Ottawa, KS, 1979.

${ }^{22}$ Ostroff, A. J., and Bacon, J.: Force and Moment Approach for Achievable Dynamics using Nonlinear Dynamic Inversion. AIAA Guidance, Navigation, and Control Conference, AIAA 99-4001, August 9-11, 1999.
${ }^{23}$ Barron Associates, Inc.: RASCLE, Robustness Analysis for Simulation-based Control Law Evaluation, Version 1.4, Design Specification, Programmer's Guide, and User's Guide. NAS1-96014, Task DC18, Jan. 2000. 